\title{
Electrokinetic Phenomena of Modified Polytetrafluoroethylene Membranes in the Oily Sewage from Oil Field
}

\author{
Aiguo LIN ${ }^{1}$, Yihua YAO ${ }^{2}$, Gang LIU ${ }^{1}$, Guozhong ZHANG ${ }^{1}$, Xiufeng LIN $^{3}$ \\ ${ }^{1}$ China University of Petroleum, Dongying, Shandong, China \\ ${ }^{2}$ Shenli Oilfield Luming Company, Dongying, Shandong, China \\ ${ }^{3}$ University of Regina, Saskatchewan, Canada \\ E-mail:shlc_zxk@yahoo.com.cn \\ Received December 12, 2008; revised March 30, 2009; accepted April 3, 2009
}

\begin{abstract}
Experimental investigations of electrokinetic phenomena of modified polytetrafluoroethylene membranes in the oily sewage from oil field were performed by using the streaming potential method. The zeta potentials of the membranes in the oily waste water are estimated on the basis of Helmholtz-Smoluchowski equation. The experiment and calculation results show that the membranes are charged negatively, whose zeta potentials maintain at around $-20 \mathrm{mV}$. And the aperture of membranes, the temperature and the filtration flux have little influence on the streaming potentials and the zeta potentials of the membranes. Also the suspended particulates in the oily sewage are charged negatively. The membranes have strong ability to withhold the suspended substance and powerful antipollution competence because of the role of the charges on the membranes.
\end{abstract}

Keywords: Oily Sewage, Membrane, Electrokinetic Phenomena, Streaming Potential, Zeta Potential

\section{Introduction}

At present, flood development is used primarily in most oilfields all over China. As the water contents of main productive zones have entered the middle or high stage, oily sewage treatment problems in nearly every oilfield are getting more and more rigorous. In order to meet the requests of sewage discharge in remote producing field and produced water reinjection in low permeable sublayer oilfields, the water quality must be handled more deeply based on the routine sewage disposal technology. Membrane separation technology is one of the most important developing tendency in deep treatment $[1,2]$. The researches on electrokinetic phenomena of membranes have attracted extensive attention in membrane science, interface science and other academic circles [3-6]. When electrolyte solution leaking porous membrane vertically(or tangentially) with definite differential pressure, potential difference will appear accordingly between two sides of the membrane, and valid electric charges on the membrane surface are usually originated from the ion absorption in solution or the liberation of some functional group on membrane micro bore surface. The existence of membrane surface charges influences the ion distribution nearby the contact interface between the membrane and the solution, and results in the formation of so-called ion diffuse electric double layer in neighboring membrane surface $[7,8]$. Electrokinetic phenomena will appear when the solution and the membrane surface move relatively as a result of pressure difference and other outside forces. However, the reports on electrokinetic phenomena of membranes in oily sewage disposal are few. Experimental investigations on electrokinetic phenomena of modified polytetrafluoroethylene membranes (The polytetrafluoroethylene membranes undergo chemical modification, hot-rolling and surface radiation treatment, which make the reprimanding oil abilities and water affinities of the membrane enhanced 
remarkably and the antipollution abilities also improved) in handling the oily waste water of oilfields are mainly performed in the paper.

\section{Experimentation}

\subsection{Experimental Apparatuses and Equipments}

Membrane fine filtration equipments. (Figure 1) under the pressure offered by centrifugal pump, oily sewage from the stuff fluid tank leak out membrane subassembly to the permeated fluid storage tank. This flow chart is divided into dead end filtration and mixed current filtration based on the way by which the stuff fluid leak the membrane (The experiments here used mixed current filtration). The membranes used in the tests are modified polytetrafluoroethylene membranes which are supplied by First Petroleum Technology Ltd., whose bore diameters are separately 0.45 and $0.8 \mu \mathrm{m}$. In the tests, the pressure differences between both sides of membrane subassembly are measured with EJA110A differential pressure transmitter (used at low pressure) and AM1151 capacitive differential pressure transmitter (used at high pressure), the measuring ranges of which are separately $0 \sim 40 \mathrm{KPa}$ and $0 \sim 300 \mathrm{KPa}$, the accuracy figures $0.1 \%$ and $0.5 \%$, and the output electric currents are both $4 \sim 20 \mathrm{~mA}$. Electric signal outputted from differential pressure transmitter are inputted to the computer by HK-PCI812 data acquisition board whose system syn- thesis error is not more than $0.2 \%$. The flow measurement of membrane assembly used glass rotameter, the main technical descriptions of which are: the measurement range $60 \sim 600 \mathrm{~L} / \mathrm{h}$, the accuracy figure $1.5 \%$. There are agitator made by self and temperature control equipment in stuff fluid tank in order to maintain the homogenization of the stuff fluid and control its temperature. To keep the sample temperature as it is collected, THD-0515W low temperature thermostatic bath was used to maintain the temperature of sample bottle, and the main technical descriptions of the bath are the measurement range $-5 \sim 100^{\circ} \mathrm{C}$, the waveness $\pm 0.05{ }^{\circ} \mathrm{C}$.

HP 34401 A digital multimeter is used to measure potential differences between both sides of membrane assembly as oily sewage leaking. WTW high accuracy inoLab 740, made in Xiamen Longlide Environment Technique exploitation Co. Ltd., is used to measure electrical conductivities of oily waste water in different temperatures. The dynamic viscosities of the sewage are tested by the concentric cylinder rotary rheometer MCR301, of which the main technical descriptions are: the minimal torque $0.1 \mu \mathrm{Nm}$, the maximum torque $200 \mathrm{mNm}$. Zetasizer3000 Zeta potential measuring instrument, made by Malvern corporation in England, could detect the electrokinetic potentials of the test solution, and the test temperatures are able to be adjusted within $10 \sim 70^{\circ} \mathrm{C}$. The paper test Zeta potentials of suspended particles in oily sewage before and after treatment with this equipment.

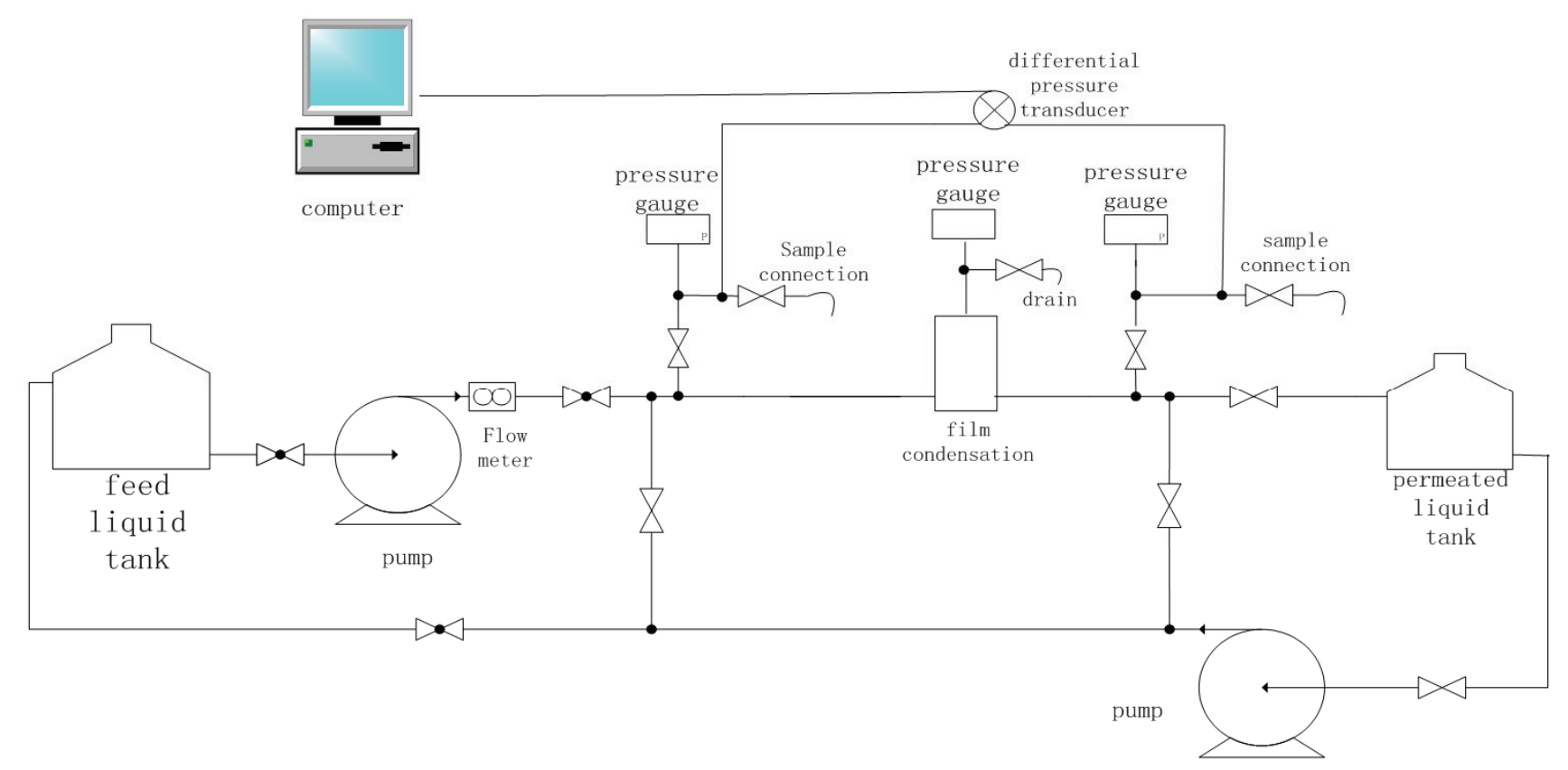

Figure 1. Membrane filtration experimental apparatus of oily sewage. 


\subsection{Test Water Samples}

The oily sewages handled in this paper are all from a sewage plant of Shengli Oilfield. In the water, suspended solid content is $10 \sim 50 \mathrm{mg} / \mathrm{L}$, total mineralization $48497.1 \mathrm{mg} / \mathrm{L}$, oil content $20 \sim 100 \mathrm{mg} / \mathrm{L}$ (measured with 721 light meter), the median diameter of suspended particles around $4 \mu \mathrm{m}$ (measured with COULTER fully automatic particle size analyzer COULTER MULTISIZER II ).

\section{Experiment Results and Analysis}

\subsection{Experiment Basis}

According to the surface and colloid science [7], potential differences between both sides of capillary pore net or porous bung made by powder compacting will appear when fluid leaking by force of compression. Millipore filter can be considered made up from lots of punctuates, and potential differences will also arise during filtering. The streaming potential is then the gradient of potential differences changing with pressure differences when electrostatic current through the membranes is zero, i.e., when solution leaking porous membrane vertically at specified pressure difference $\Delta P$, there will be a potential difference $\Delta E$ between both sides of membrane accordingly, and the streaming potential can be described as:

$$
v=\mathrm{d}(\Delta \mathrm{E}) / \mathrm{d}(\Delta \mathrm{P})
$$

The streaming potentials of membranes can be obtained by tested directly, in which, just connect a voltmeter between the two ends [9]. The streaming potentials can only describe the membrane charged properties qualitatively, but not the quantitative magnitude which can be indicated with Zeta potentials of the membrane surface. The double electrode layer theory considers that there is a "glide surface", on which the solution and surface move relatively, at some distance away from the surface within the membrane surface ion diffuse electric double layer, and the potential difference between "glide surface" and main part of the solution is called Zeta potential. Zeta potential of the membrane surface is an important parameter which determines electrokinetic phenomena of membranes, and depends on membrane material and solution system intently [10]. The micro bore surface Zeta potential ( $\zeta$ ) [3] of modified polytetrafluoroethylene membranes can be estimated by outstanding Helmholtz-Smoluchowski equation using membrane streaming potential which has been obtained:

$$
v=\frac{d(\Delta E)}{d(\Delta P)}=\frac{\varepsilon_{0} \varepsilon_{r} \zeta}{\mu k} \Rightarrow \zeta=\frac{v \mu k}{\varepsilon_{0} \varepsilon_{r}}
$$

In which, $v$ is the streaming potential, whose unit is $\mathrm{V} / \mathrm{Pa} ; k$ the electric conductivity of solution, unit $\mathrm{S} / \mathrm{m}\left(1 S=1 c \cdot s^{-1} \cdot V^{-1}\right) ; \mu$ the viscosity, unit $P a \cdot s, \varepsilon_{r}$ and $\varepsilon_{0}$ are separately relative dielectric constant and vacuum dielectric constant, whose units are $\mathrm{F} / \mathrm{m}$ $\left(1 F=1 c \cdot V^{-1}\right)$.

Helmholtz-Smoluchowski equation is suit for the case when the pipe diameter or capillary tube diameter is far larger than the thickness of double electrode layer. The thicknesses of double electrode layer of membrane borehole wall are nanometer degree, while the bore diameters of modified polytetrafluoroethylene membranes used in the experiments are meter degree, in which the latter are far larger than the former, so the equation is fit for the estimate of the membrane surface Zeta potentials here.

\subsection{Membrane Surface Zeta Potentials}

As the membrane bore diameters, filtering temperatures, filtering flow rates and other elements have obvious effects on filtering effect, and those elements also probably influence the membrane surface Zeta potentials, filtering tests with membranes of different bore diameters were performed under different temperatures and specified flow rates. The potential differences of oily waste water between both sides of the modified PTFE filter membrane are measured with HP 34401A digital multimeter at correspondent operating conditions. The streaming potential $v$ of the membrane at different operating conditions could be calculated according to Formula (1) with the potential difference $\Delta E$ and the pressure difference $\Delta P$ obtained from experiments. Some results are shown in Table 1.

The table shows that, although there are differences among the membrane bore diameters and operating conditions, the changes of membrane streaming potentials are not obvious: of the 9 tests, the average maximal value is $-5.01 \mathrm{mV} / \mathrm{KPa}$, the maximal value $-5.3 \mathrm{mV} / \mathrm{KPa}$, the minimum value $-4.9 \mathrm{mV} / \mathrm{KPa}$ and the standard deviation $0.13642 \mathrm{mV} / \mathrm{KPa}$. In fact, the absolute value of membrane streaming potentials is probably affected by the electrolyte concentration of solution, types and valence states of basic ion and negative ion and other elements [6]. It is found that the oily waste water investigated here contains $\mathrm{Na}^{+}$with a concentration of $15951.58 \mathrm{mg} / \mathrm{L}$ and $\mathrm{Mg}^{2+}$ of only $261.91 \mathrm{mg} / \mathrm{L}$ through water quality analysis, so the effect of $\mathrm{Na}^{+}$is dominating, and as its concentration is far larger than the critical concentration given in reference [6], the components of the waste water would not change suddenly. Thus the slight alternation of ion concentration caused by sample collection and other reasons would not influence the value of membrane 
Table 1. Membrane streaming potentials at different operating conditions.

\begin{tabular}{ccccc}
\hline Sequence number & Bore diameter of membrane $(\mu \mathrm{m})$ & Temperature $\left({ }^{\circ} \mathrm{C}\right)$ & Given flow rate $(\mathrm{L} / \mathrm{h})$ & $\begin{array}{c}v \\
(\mathrm{mV} / \mathrm{Kpa})\end{array}$ \\
\hline 1 & 0.8 & 30 & 600 & -4.9 \\
2 & 0.8 & 50 & 400 & -5.1 \\
3 & 0.8 & 70 & 250 & -5.0 \\
4 & 0.45 & 30 & 400 & -4.9 \\
5 & 0.45 & 50 & 250 & -4.9 \\
6 & 0.45 & 70 & 600 & -5.0 \\
7 & 0.45 & 30 & 250 & -5.3 \\
8 & 0.45 & 50 & 600 & -4.9 \\
9 & 0.45 & 70 & 400 & -5.1 \\
\hline
\end{tabular}

Table 2. Conductance ratios and dynamic viscosity of oily waste water under different temperatures.

\begin{tabular}{lccc}
\hline Temperature of water sample $\left({ }^{\circ} \mathrm{C}\right)$ & 30 & 50 & 70 \\
\hline Conductance ratio $(\mathrm{S} / \mathrm{m})$ & 2.5 & 3.5 & 4.9 \\
\hline dynamic viscosity $(\mathrm{mPa} \cdot \mathrm{s})$ & 0.944 & 0.689 & 0.544 \\
\hline
\end{tabular}

Note: the conductance ratios shown in the table were measured by inoLab 740, of which the measuring range is LR001 and the accuracy degree is $0.001 \mu \mathrm{S} / \mathrm{cm}$.

Table 3. Dynamic viscosities factors $\mu$ of oily waste water under different temperatures.

\begin{tabular}{cccc}
\hline Temperature of water sample $\left({ }^{\circ} \mathrm{C}\right)$ & 30 & 50 & 70 \\
\hline Dynamic viscosity $(\mathrm{mPa} \cdot \mathrm{s})$ & 0.944 & 0.689 & 0.544 \\
\hline
\end{tabular}

According to the data above, Zeta potentials of modified polytetrafluoroethylene membranes can be calculated through Formula (2). Table 4 shows Zeta potentials of the membrane surface under different experiment conditions.

streaming potentials, which is accordant to the experiment results shown in Table 1.

It has been told before that the membrane streaming potentials can not indicate the magnitude of membrane surface electrical properties quantitatively. In order to estimate the membrane surface Zeta potentials and quantitatively describe the electrical properties, HelmholtzSmoluchowski equation is needed. The correlating parameters in the equation are defined as shown in Table 2 and Table 3 by experiments.

It is thus evident that the Zeta potentials of modified polytetrafluoroethylene membrane surface are between $-18.67--21.94 \mathrm{mV}$, of which the average value is $-20.06 \mathrm{mV}$ and the standard deviation 1.3078. In other words, during the oil sewage treatment using modified polytetrafluoroethylene membrane, the membrane sur- face charged negatively and the influences on membrane surface Zeta potentials caused by membrane bore diameters and operating conditions are not obvious. The Zeta potentials of membrane surface are maintained around $-20 \mathrm{mV}$.

Table 4. Zeta potential on the modified PTFE membrane under different conditions.

\begin{tabular}{cccc}
\hline No. & Zeta/mV & No. & Zeta/mV \\
\hline 1 & -18.67 & 6 & -21.52 \\
2 & -19.85 & 7 & -20.19 \\
3 & -21.52 & 8 & -19.07 \\
4 & -18.67 & 9 & -21.94 \\
5 & -19.07 & & \\
\hline
\end{tabular}


Table 5. Zeta potential of suspended particles under different temperatures.

\begin{tabular}{ccc}
\hline Sequence number & $\begin{array}{c}\text { Temperature } \\
\left({ }^{\circ} \mathrm{C}\right)\end{array}$ & $\begin{array}{c}\text { Zeta potential } \\
(\mathrm{mV})\end{array}$ \\
\hline 1 & 30 & -14.2 \\
2 & 40 & -1.8 \\
3 & 50 & -4.4 \\
4 & 60 & -8.2 \\
5 & 70 & -12.3 \\
\hline
\end{tabular}

\subsection{Zeta Potentials of the Suspended Particles in Oily Waste Water}

In order to profoundly study the electrokinetic phenomena of modified polytetrafluoroethylene membranes, the charging properties of suspended particles should be known. As the electrical properties of general colloidal particles are not expressed with the value of electric charges but the Zeta potential $\zeta$, the paper measured Zeta potentials of suspended particles under various temperatures by Zetasizer 3000 Zeta potential test set, and the results are shown in Table 5 .

Obviously, the Zeta potentials are all negative and of the same electrical properties as the surface Zeta potentials. Zeta potential changes apparently as temperature changes, but not show regular patterns of increasing or decreasing monotonously, which is probably relevant with the vergent suspended particles and the fact that sampling sewage could not be absolute uniform.

Seen from the separation behavior, conventional membrane separation filtration is based on a physical sieving rule, that is, the membrane allows the components with smaller bore diameters to go through but entraps those with larger or similar bore diameters. Obviously, with the decrease of the particle diameters, the membrane bore diameters should decreases correspondently, which is bound to cause such problems as decreasing flux and increasing operating costs. However, besides physical sieving, the charging membrane has Donnan effect. According to the Donnan balance model, when the solution leak through membrane with charging group, the concentration of counter ions(those ions taking opposite charges to that fixed in the membrane) in the membrane is larger than that in the solution body, while the behavior of ions with same charges is just opposite. Donnan potential difference is thus formed and prevents the ions with same charges in the solution body from diffusing to the membrane. In order to maintain electrically neutral, the membrane also entraps counter ions. So such charging membrane has particular adsorptive separation properties. Additionally, charging groups generate in the membrane, which strengthens the hydrophilicity and increases the perme- ability of the membrane. Meanwhile, the antipollution competence of the membrane is improved markedly because of the charge effect that charges with the same electric nature reject each other.

Thus the modified polytetrafluoroethylene membranes have strong ability withholding the suspended substance and powerful antipollution competence as a result of the role of the charges on the membranes when used to handle oily waste water, which prolong the backwashing cycle and service life of the filter element. The content about the effectiveness of the modified polytetrafluoroethylene membranes processing oily sewage will be elaborated in other papers.

\section{Conclusions}

When dealing with oily waste water, the streaming potentials of the modified polytetrafluoroethylene membranes maintain around $-5 \mathrm{mV} / \mathrm{KPa}$ while the membrane surface Zeta potentials around $-20 \mathrm{mV}$, which are slightly dependent on such operating conditions as membrane bore diameters, flow rates, etc. The Zeta potentials of the suspended particles in the sewage being processed are also negative.

The membranes have strong ability withholding the suspended substance and powerful antipollution competence because of the role of the charges on the membranes.

\section{References}

[1] A. G. Lin, P. Y. Liu, G. Liu, and G. Z. Zhang, "Progresses of membrane separation technique in oil-bearing water treatment in oil fields," Industrial Water Treatment, Vol. 26, No. 1, pp. 5-8, 2006.

[2] Q. X. Zou and Z. Y. Lu, "Survey of oil-field waste water treatment," Industrial Water Treatment, Vol. 21, No. 8, pp. 1-3, 2001.

[3] W. R. Bowen and X. W. Cao, "Electrokinetic effects in membrane pores and the determination of zeta-potential," Journal of Membrane Science, Vol. 140, pp. 267-273. 1998.

[4] A. Szymczyk and P. Fievet, "Characterization of surface properties of ceramic membranes by streaming and membrane potentials," Journal of Membrane Science, Vol. 146, pp. 277, 1998.

[5] J. M. M. Peeters, "Characterization of nanofiltration membranes by streaming potential measurements," Colloids and Surface A: Physicochemical and Engineering Aspects, Vol. 150, No. 1-3, pp. 247-259, 1999.

[6] J. Wang and X. L. Wang, "Electrokinetic phenomena of poly olefin microporous membranes in the electrolyte solutions studied by using the streaming potential method," Journal of Chemical Engineering of Chinese Universities, Vol. 17, No. 4, pp. 372-376, 2003.

[7] Z. Shen and G. T. Wang, "Surface and colloid science [M]," Chemical Industry Press, 1997.

[8] S. Sdukhin and B. V. Derjaguin, "Surface and colloid science [M]," London: Wiley - Interscience Publication, 1974. 
[9] M. F. Zhu, C. Y. Gong, and J. Y. Su, "Experimental method of the streaming potential of charge modified microporous membrane," Chinese Medical Equipment Journal, Vol. 1, pp. 1-4, 1996.
[10] M. Nystrom and M. Lindstrom, "Streaming potential as a tool in the characterization of ultrafiltration membranes," Colloid Surface, Vol. 36, pp. 297, 1989. 